\title{
THE ASPECT OF NEUROCOGNITIVE AND REHABILITATION ON ALEXIA WITHOUT AGRAPHIA CASE
}

\author{
KETUT WIDYASTUTI, AAA PUTRI LAKSMIDEWI, PUTU EKA WIDYADHARMA* \\ Department of Neurology, Faculty of Medicine, Udayana University, Sanglah General Hospital, Bali, Indonesia. \\ Email: eka.widyadharma@unud.ac.id
}

Received: 16 October 2017, Revised and Accepted: 13 November 2017

\begin{abstract}
Alexia without agraphia is a syndrome of disconnection which causes an abruption of visual input to language area. Pure alexia can impact reading ability and create dependency on patients when they do their daily work. An early stage of alexia without agraphia can be detected through a set of neurocognitive evaluation. Besides, it is believed that this assessment has a significant role in anticipating the lag of a proper rehabilitation. Herein, we report a case of a 45-year-old female; she complained about having a visual malaise on her right vision and also said to lose her ability to read very suddenly 14 days prior. On language diagnostic screening, the patient was able to speak fluently, could comprehend and repeated phrases regularly but an experienced loss of color recognition. The patient's visual acuity seemed reasonable and was able to write words or phrases in spite of unable to read. The patient suffered from stage 2 hypertension, hypertriglyceridemia, and diabetes mellitus according to physical examination and laboratory evaluations. Furthermore, an acute infarction on the left posterior cerebral artery which involved the splenium of the corpus callosum and left visual cortex was detected on brain magnetic resonance imaging. Furthermore, the cerebral magnetic resonance angiography results did not show any hemodynamic disruption occurred by occlusion or stenosis. A tactile-kinesthetic exercise and multiple oral re-reading (MOR) program had increased the rehabilitation efficacy for reading ability. However, right-sided homonymous hemianopia did not demonstrate any significant improvements. Alexia without agraphia can be a secondary symptom which occurs in stroke patients, due to the lesion in occipital cortex and area of the posterior corpus callosum on the dominant hemisphere. Neurocognitive evaluation by language diagnostic modalities on alexia without agraphia had demonstrated reading disruption, despite intact writing, and other language-related abilities. Tactile-kinesthetic exercise and MOR were reported to improve the malaise of reading ability.
\end{abstract}

Keywords: Alexia without agraphia, Stroke, Neurorehabilitation, Neurocognitive.

(C) 2018 The Authors. Published by Innovare Academic Sciences Pvt Ltd. This is an open access article under the CC BY license (http://creativecommons. org/licenses/by/4. 0/) DOI: http://dx.doi.org/10.22159/ajpcr.2018.v11i2.23150

\section{INTRODUCTION}

Cognitive disorders can be caused by various brain problems, including trauma, tumors, and cerebrovascular diseases such as strokes and genetic factors such as Alzheimer disease [1] and complication of viral brain infections in immunocompromised patients with HIV/AIDS [2,3]. Evidence suggests that metabolic syndrome and associated factors such as visceral obesity, elevated triglycerides, elevated fasting blood glucose, high blood pressure, and decreased high-density lipoprotein all have detrimental effects on cognition [4]. Stroke is increasingly recognized as an essential cause of cognitive problems. The prevalence of cognitive impairment after stroke is high, and their combined effects significantly increase the cost of care and health resource utilization, with reflections on impairment of patient's functional ability and increased morbidity and mortality rates [5].

The most common types of cognitive deficits arising from stroke are disturbances of attention, language disorders, delayed recall, and executive dysfunction. Language disorders in stroke patients are very complicated regarding clinical and classification, include aphasia, Alexia, agraphia, and acalculia. Regardless of the fact that a significant number of these disorders spontaneously improve, it is necessary to start treatment as early as possible. Early rehabilitation of language disorders is essential because it is crucial for everyday communication and can be done in conjunction with motoric rehabilitation $[5,6]$.

Writing and reading skills are a part of language-related ability that possesses a significant role in our daily communication and interaction. Alexia without agraphia refers to the disruption of reading and writing ability due to brain damage and is not manifested by blindness, paralysis, and other neurologic deficits. Alexia without agraphia is commonly found on most types of aphasia syndrome [6]. Pure alexia without agraphia is diagnosed based on the form of reading ability disruption which contradicts with patient's writing ability remains normal. Neurocognitive analysis on alexia without agraphia based on clinical performance is delivered to anticipate the lag of conducting proper rehabilitation [7]. The following report is a case of pure alexia without agraphia from the perspective of neurocognitive and recovery analyses aspects which should be considered as necessary on aphasia syndrome.

\section{CASE REPORT}

Informed consent was taken from the patient. A 45-year-old female who worked as a hotel employee complained about the decrease in her right vision and followed by a sudden reading difficulty since 14 days prior. She retained the ability to speak, understood instructions and was able to repeat phrase but hardly able to re-read words or sentences she wrote. The patient was able to comprehend the nature, and use of a subject presented visually and recognized faces. She had hypertension and diabetes history and had been using insulin since 5 years ago. There was no suggestive history of heart disease, trauma, blood transfusions, joint pain, rashes on skin, photosensitive, or seizure activity. The patient also had no record of losing consciousness, fever, headache, vomiting, voice problem, or walking weakness.

Based on physical examination, it was found that she had a stage two hypertension with a measured blood pressure of 170/100 $\mathrm{mmHg}$. She had normal motoric and sensory responses, as well as upper-lower extremities reflex responses. A Mini-Mental State Examination was given for measuring her neurocognitive functions in which she gained a total score of 25 , with failures on recall domain, copying image, reading, 
and writing. Language modalities examination displayed average speaking ability, understanding, and good phrase repetition, but failed to name colors. She had reading difficulties, both for words and phrases, but retained excellent writing ability.

Ophthalmic evaluation revealed right hemianopia with intact visual acuity, pupillary functions, and eye movements. Pre-prandial blood sugar levels, hemoglobinA1c, triglyceride, and total cholesterol levels were $189 \mathrm{mg} / \mathrm{dL}, 8.06 \%, 348 \mathrm{mg} / \mathrm{dL}$, and $214 \mathrm{mg} / \mathrm{dL}$, respectively. Furthermore, brain magnetic resonance imaging demonstrated acute infarction on left posterior cerebral artery which perfuses the splenium of the corpus callosum and left visual cortex (Fig. 1). Furthermore, cerebral magnetic resonance angiography demonstrated occlusion on left posterior cerebral artery (Fig. 2).

Treatments recommended for this patient were outpatient treatment along with therapy to control the risk factors such as hypertension, diabetes, and dyslipidemia, also advised to have efforts for preventing stroke by giving patient platelet anti-aggregation and improved lifestyle. Rehabilitation to improve reading ability suggested by providing a tactile-kinesthetic exercise and multiple oral re-reading (MOR) as it can contribute to enhanced letter identification and reading capacity and speed in general. The right homonymous hemianopia still existed and showed no significant improvement.

\section{DISCUSSION}

It is reported that alexia without agraphia is a rare case. Pure alexia without agraphia is also known as pure alexia, pure word blindness, agnostic alexia, occipital alexia, posterior alexia, verbal alexia, or letter-by-letter reading is disconnection syndrome associated with lesion or infarction on the left occipital cortex and posterior part of the corpus callosum $[7,8]$. In this case, the patient lost her ability to read words or phrases albeit she was able to spell letter-by-letter. She experienced difficulty on copying word by word compared to automatic writing.

Patient's reading ability was not entirely degenerated; she was in fact still able to spell letter-by-letter in words. Its level of deficit contributes to this reading disability in patients with Alexia. It is discovered that on a mild case it only affects the speed of reading, while on a more severe case, the patient will experience a problem with identifying phrases and even letters [7]. As we all believe that reading and writing are some main components of our language ability, a deficit in one or more of these capabilities will thus potentially impair language comprehension and expression. The language area is lying along fissure of Sylvian/ lateral sulcus of the categorical hemisphere. Visual information is received by the retina and passed to the lateral geniculate nucleus of the thalamus and carried to the visual cortex (area 17 and 18) and angular gyrus on Wernicke area to process auditory and visual information. The information is then delivered to Broca area through arcuate fasciculus to generate a detailed and coordinated vocalization pattern. The projection of this model is in turn sent to speech articulation on insula toward motoric cortex to correct lips, tongue, larynx movement, and ultimately producing voice [9].

The activity of writing involves a complex coordination of several systems comprising the motoric, linguistic, and visual-kinetic system. Exner's center is an area responsible for the writing activity. It lies in the middle of the frontal gyrus of the dominant hemisphere. There is a path of substantia alba which supports bridging from Wernicke area and Exner's center area [10]. In the parietal lobe, this area is responsible for the formation of written words, in which, by means of the connection with Wernicke area, it is then transformed to become grapheme before being transferred to the lateral convexity of the left frontal lobe where Broca and Exner's area play their part in changing it to become motoric expression [11].

Patient in this report suffered from right hemianopia and had difficulty in naming colors. The lesion on left occipital cortex results in right hemianopia. The injury leads to no visual information received from the left visual field and leaving only the right occipital to process the visual information and then crosses to corpus callosum to the left posterior hemisphere for the graphics decoding process. Patient with a lesion on splenium of the corpus callosum or the area of substantia alba will not be able to read or name colors due to no visual information reaches left language area, but it will not face difficulty on writing/copying words because visual details to activate left motoric area through corpus callosum allocated more anterior. The natural writing ability and copying words are still intact due to language area including angular gyrus, Wernicke, Broca, and left motoric cortex are still intact and connected. However, afterward, the patient could not re-read whatever she just wrote [12].

The Alexia without agraphia, in this case, was associated with the secondary impact of stroke which caused an infarction on the area of the left occipital. Some Alexia without agraphia cases shows the relation with occipital lobe, in addition to the disorder of color identification. Alexia without agraphia is a result of the indirect involvement of angular gyrus and left hemisphere which has a significant role in recognizing words. Therefore, the reading ability becomes affected. Most cases are caused by occlusion of the left posterior cerebral artery which leads to an infarction on left visual cortex and corpus callosum splenium, which was found in this case. The involvement of splenium disrupts the connection between right visual cortex which is still intact with left angular gyrus as the cortical center for reading ability. The standard writing ability is attributed to the entire left angular gyrus, which obtains its vascular supply from the middle cerebral artery, as it is the connection pathway that is destroyed with splenium infarction and not an angular gyrus itself [7].

A short screening could be executed to identify the existence of Alexia. Hence, a complete assessment is necessary to determine the precise area which has a disorder, followed by an efficient therapy program. The evaluation itself involves an assessment toward reading comprehension

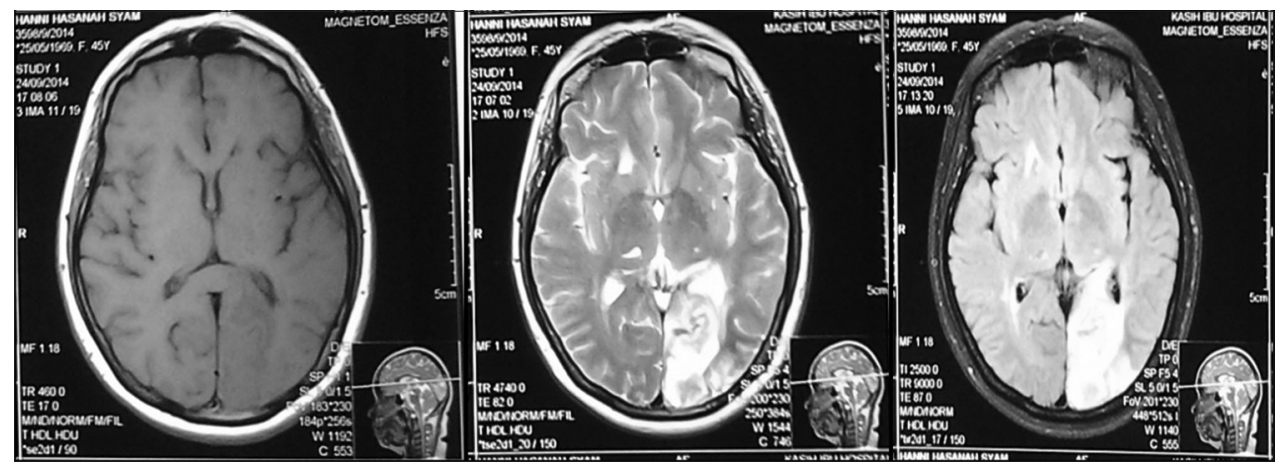

Fig. 1: Brain magnetic resonance imaging revealed acute infarction on left posterior cerebral artery which perfuses the splenium of the corpus callosum and left visual cortex 


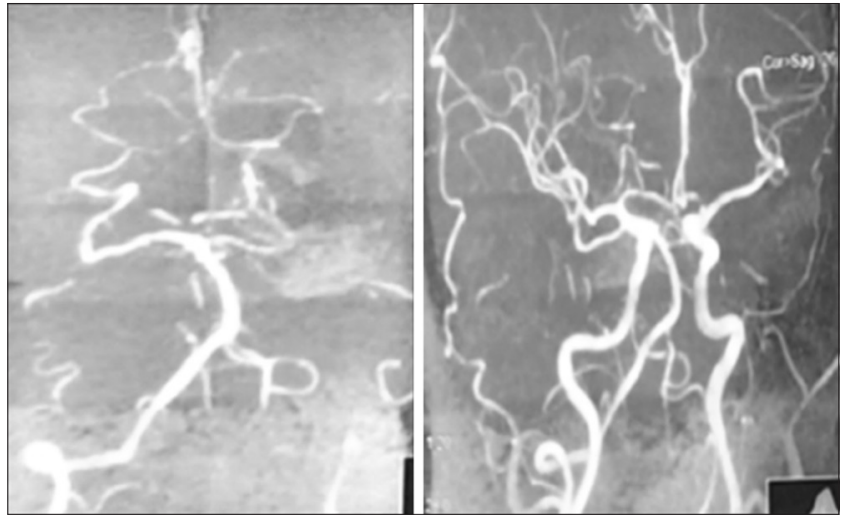

Fig. 2: Cerebral magnetic resonance angiography revealed occlusion on left posterior cerebral artery

to determine the level of lesion, analyzing single word reading ability and determining the area of strength and weakness of visual, writing, naming, and spelling-related abilities [11].

All of the aphasia-related tests require a reading comprehension sub-test to evaluate semantic meaning ability. A reading comprehension begins with letters, a letter recognition and matches the written letter with a various shape of letters. Furthermore, on words level, for example, naming a name of cities or countries, followed by phrase level, at the end of assessment patient needs to read the whole paragraph of one topic and answers the questions related to the content. Prompt examination for the reading disorder is to determine its level of disability, whether it is in syllables, words, phrases, sentences, or paragraphs level. Tasks are given to patient such as: Pairing a letter, pairing a word with an image, compiling words based on their category, antonym, synonym, and matching a word with its definition. Completing phrases or sentences, follows written instruction, answering yes or no questions related to a sentence, or a paragraph [11]. A cognitive process of writing works in parallel with the reading process. An examination for the capability of writing is possibly done through the reading test as well as by giving the patient to write down words that are read aloud. There is no time restriction for the patient; furthermore, there will be an evaluation of the results in the area of addition, transposition, substitution, and phonology [13].

In each case of pure Alexia, there are many ways of how to handle the patient; it depends on the level of the patient's reading impairment, the length of onset, and other relevant gaps. To date, there is no practical therapy to reverse patient's normal reading ability or read fluently; nevertheless, an exercise can be given to improving letter identification or reading speed [7]. A tactile-kinesthetic training can be implemented on the level of word impairment by attempting to write a letter on the palm or using a finger to recognize letter visually, while a deficit on the phrase level can be rehabilitated using MOR technique. MOR is usually delivered by reading text out loud repeatedly and measure the reading speed and its accuracy until it reaches its reading target. This exercise is recommended to be applied for at least 30 min daily or 3-5 times daily. The patient can be assisted initially, but they can perform it independently later on [14]. Most sentences consist of frequently used words so that the patient is given an amount of words to be recognized (usually around 125-150 words) of which all of them are often used [7].

\section{CONCLUSION}

Alexia without agraphia is a syndrome caused by a lesion on the left occipital cortex and left posterior of corpus callosum splenium which leads to a loss of visual input to language area and often accompanied with hemianopsia. Pure Alexia possibly coexists with or without color blindness and visual object agnosia. In the meantime, there is no therapy believed to assist the patient to be able to read regularly, but rehabilitation efforts may be able to ameliorate disability and contributes progress. A tactile-kinesthetic exercise and MOR can be advocated to improve letter identification or reading ability/speed.

\section{AUTHOR CONTRIBUTIONS}

This work was carried out in collaboration between all authors. Author KW designed the study and perform neuro-cognitive assesment, AAAPL managed the patient in Neuro-behavior Clinic, PEW wrote the first draft and editing of the manuscript. All authors read and approved the final manuscript.

\section{CONFLICTS OF INTERESTS}

The Authors declare that they have no conflicts of interests.

\section{REFERENCES}

1. Kaur K, Kaur R, Kaur M. Recent advances in Alzheimer's disease: Causes and treatment. Int J Pharm Pharm Sci 2016;8:8-15.

2. Widyastuti K, Adnyana MO, Sudewi AA. Low Nadir and Current CD4 Account as a risk factor for Cognitive Impairment in HIV Patient in Sanglah General Hospital Denpasar 2012;29:28-35.

3. Widyadharma E, Satiti S, Nuradyo D, Setyopranoto I, Wijayanti Y. The difference of CD4 count between HIV positive patients with cognitive decline and without cognitive decline. Biomed Pharmacol J 2017;10:969-78.

4. Nerkar D, Mukherjee A, Mehta BK, Banerjee S. Metabolic syndrome associated complications. Int J Pharm Pharm Sci 2015;7:22-5.

5. Sun JH, Tan L, Yu JT. Post-stroke cognitive impairment: Epidemiology, mechanisms and management. Ann Transl Med 2014;2:80.

6. Henderson VW. Alexia and agraphia. In: Finger S, Boller F, Tyler KL, editors. Handbook of Clinical Neurology. Vol. 9. Stanford: Elsevier; 2010. p. 583-601.

7. Starrfelt R, Olafsdóttir RR, Arendt IM. Rehabilitation of pure alexia: A review. Neuropsychol Rehabil 2013;23:755-79.

8. Ardila A. Aphasia Handbook. Miami, FL: Florida International University; 2014. p. 102-35.

9. Barret K, Barman SM, Boitano S, Brooks HL. Ganong's Review of Medical Physiology. 24 $4^{\text {th }}$ ed. New York: McGraw Hill; 2012. p. 283-95.

10. Campbell WW. Dejong's the Neurologic Examination. $7^{\text {th }}$ ed. Philadelphia, PA: Lippincott Williams \& Wilkins; 2013. p. 87-111.

11. Sinanovic O, Mrkonjic Z, Zukic S, Vidovic M, Imamovic K. Poststroke language disorders. Acta Clin Croat 2011;50:79-94.

12. Cumming JL, Mega MS. Neuropsychiatry, and Behavioral Neuroscience. New York: Oxford University Press; 2003. p. 70-96.

13. Sheldon CA, Malcolm GL, Barton JJ. Alexia with and without agraphia: An assessment of two classical syndromes. Can J Neurol Sci 2008;35:616-24

14. Kim ES, Lemke SF. Behavioural and eye-movement outcomes in response to text-based reading treatment for acquired alexia. Neuropsychol Rehabil 2016;26:60-86. 